\title{
Three-dimensional Cat Virtual Anatomy: Development of an Interactive Virtual Anatomical Software
}

\author{
Juan Sebastián Osorio-Echeverri ${ }^{1}$ Diana Alexandra Orrego-Metaute ${ }^{2}$ Juan Pablo Murillo-Escobar ${ }^{2}$ \\ Lynda Tamayo-Arango ${ }^{1}$
}

${ }^{1}$ CIBAV Research Group, Faculty of Agrarian Sciences, University of Antioquia, Medellín, Colombia

2 Biomedical Research and Innovation Group, Faculty of Exact and Applied Sciences, Metropolitan Technological Institute, Medellín, Colombia

Address for correspondence Lynda Tamayo-Arango, VM, PhD, CIBAV Research Group, Faculty of Agrarian Sciences, University of Antioquia, 65-87, Medellín, Colombia (e-mail: lynda.tamayo@udea.edu.co).

J Morphol Sci 2019;36:105-114.

\begin{abstract}
\section{Keywords}

- three-dimensional anatomy

- education in anatomy

- multimedia anatomy resources

Background Three-dimensional (3D) virtual models are novel tools to teach veterinary anatomy.

Objective The aim of the present study was to create a 3D cat image software and a library of cross-sectional images.

Methods Modeling of the 3D cat organs and structures was done with Autodesk Maya, version 2017 (Autodesk Inc., San Rafael, California, USA) and ZBrush, version 4R7 (Pixologic, Los Angeles, CA, USA) software. In order to obtain the images for the library, three cadavers of adult cats were used, with the following techniques: 1 ) scanning by magnetic resonance imaging (MRI) at 3-mm intervals, 2) scanning by computed tomography (CT) at 2-mm intervals, and 3) photographing of 178 transverse cuts at 2.5-mm intervals from the frozen cadavers. Out of all the images, thirty images of each technique were selected. An interactive software was developed with the modeled 3D cat and the selected images using Unity, version 5.4 (Unity Technologies, San Francisco, CA, USA).

Results A virtual 3D cat model was obtained with 418 labeled structures of the skeletal, muscular, circulatory, nervous, respiratory, digestive, urinary, and integumentary systems. The virtual interface enables the manipulation of the 3D cat in all views and the visualization of the selected images in a chosen localization along the body of the cat. The library of images allows comparison among CT, MRI and photographs of transverse cuts.

Conclusions The software interface facilitates the access to the content for the user. Sectional images of the cat and of its body structures can be easily understood. This new 3D software of cat anatomy is another tool that can be used in teaching veterinary anatomy.
\end{abstract}

\section{Introduction}

Virtual models are interesting tools that show anatomy in a hyper-realistic way through three-dimensional (3D) virtual objects representing anatomical structures. ${ }^{1-5}$ Compared with static images or videos, 3D virtual objects allow the creation of realistic and interactive virtual spaces. ${ }^{3-5}$

These models allow taking out parts or systems of the body, such as the skeleton, organs, or vascular structures, virtually mimicking a body dissection. The user can visualize and manipulate the structures according to their interest, even beyond what reality allows. ${ }^{5-8}$ In addition, a large number of structures and organs can be shown in a simplified, clear, and illustrative way, due to interfaces that facilitate their display and manipulation according to the particular interest of the user. ${ }^{4-7}$ In this respect, the use of virtual models is a new tool that facilitates the understanding of the localization of anatomical structures. ${ }^{8-10}$ received

August 2, 2018

accepted

February 11, 2019
DOI https://doi.org/

10.1055/s-0039-1683964. ISSN 2177-0298.
Copyright $\odot 2019$ by Thieme Revinter

Publicações Ltda, Rio de Janeiro, Brazil
License terms

(c) $(1) \$$ 
It is important to highlight the quality and realism of 3D models compared with traditional anatomical drawings, and the possibility of viewing from several angles for easier interpretation and understanding. ${ }^{4,5,8,11}$ Additionally, these models allow the simulation or the visualization of physiological and dynamic processes. ${ }^{11-13}$ For example, recreating natural processes such as the heartbeat or the movement of the body or of a limb in a virtual way allows analyzing them in more detail. ${ }^{13,14}$

The increasing use of advanced imaging diagnostic techniques such as computed tomography (CT) and magnetic resonance imaging (MRI) in veterinary medicine, especially in small animal practice, requires a deep knowledge of anatomy. ${ }^{6,8,15}$ Interactive virtual models based on crosssections, MRI, and CT images facilitate the understanding of the anatomy and, consequently, a better interpretation of the image for future diagnosis purposes. ${ }^{10,15}$

The complexity of 3D models requires the development of navigation systems that provide fast and dynamic exploration of scenes. ${ }^{1,4,5,13}$ User interface approaches facilitate a precise composition of the scene and its exploration according to the conditions considered appropriate. In addition, the animation combined with the interaction facilitates the rapid exploration of complicated scenes. ${ }^{4-7}$

In human anatomy, multiple virtual 3D models of the body have been developed to support teaching in a detailed way, allowing the development of simulators of physiological cycles. ${ }^{11-13,16-20}$ In veterinary medicine, several models that cover the entire body of the animal have been developed for dogs and mice. ${ }^{14,21-23}$

The aim of the present work was to develop an interactive 3D cat virtual model and an image library using transverse MRI, CT, and photograph images of the entire body of the cat.

\section{Materials and Methods}

\section{Ethics}

The present study was approved by the Ethics Committee for Animal Experimentation of the Universidad de Antioquia (CEEA-03/10/2014).

\section{Preparation of the Cadaver}

A fresh cadaver of an adult $4 \mathrm{Kg}$ male mongrel cat was donated to the Hospital Veterinario de la Universidad de Antioquia. The cause of death was an acute infectious process that did not affect its anatomy. It was kept refrigerated (between 4 and $0^{\circ} \mathrm{C}$ ) for $\sim 10$ hours, while the initial positioning and freezing process was started. No fixation or vascular perfusion with any substance was performed to avoid any alteration in the anatomy. The cadaver was prepared as follows: positioned in left lateral decubitus with the head slightly elevated on a blanket above the level of the trunk, the forelimbs parallel and flexed at the humeral, ulnar and carpal joints; similarly, both hind limbs were parallel and flexed at the hip, stifle and tarsus joints. Finally, the tail was flexed laterally, parallel to the trunk ( - Fig. 1A). The cadaver was frozen at $-20^{\circ} \mathrm{C}$ for 48 hours. An open wooden box was wrapped with polystyrene ( - Fig. 1B), filled with $\sim 2 \mathrm{~cm}$ of embedding fluid ( $94.5 \%$ water, $5 \%$ gelatin, $0.5 \%$ methylene blue), and put into a deep freezer at- $20^{\circ} \mathrm{C}$. When the fluid was frozen, the cadaver was placed inside the box ( - Fig. 1C), and embedding fluid was added until $50 \%$ of the body was covered to be frozen again. Finally, the remaining space of the box was completed with embedding fluid and frozen at $-20^{\circ} \mathrm{C}$ for 1 week. A frozen block of embedding fluid was obtained with the cadaver positioned inside (-Fig. 1D).

\section{Sectioning of the Cadaver}

A band saw (Generic butcher cutter band) was used for the cutting process. The band saw had a load tray, adjustable stop, guides for the band, and rubber feet. The load tray had a raised edge on one of its sides and moved on a rail in the direction of the saw, the adjustable stop allowed to limit the cutting space as desired, the guides for the band kept the position of the cutting blade stable preventing displacements and keeping the cut on the same plane, and the rubber feet were non-slip to avoid machine movements that deflect the band at the time of cutting. The band used was a $27 \mathrm{~mm}$ wide, $1.6 \mathrm{~mm}$ thick steel blade (Baileigh Industrial, California, USA), with 4 teeth per inch of length. The sectioning process was made from cranial to caudal, making serial cross-sections of the animal with a thickness of $2.5 \mathrm{~mm}$ between cuts. The frozen block was removed from the wooden box, inside the polystyrene. Before starting the sectioning, and using a digital caliper (Mitutoyo Corporation, Tokio, Japan) (accuracy of $0.01 \mathrm{~mm}$ ) to measure the distance, the adjustable stop was fixed to a space of $2.5 \mathrm{~mm}$ from the cutting blade to fix the cuts to this thickness. Then, the frozen block was carefully placed on the load tray, keeping the left side of the face resting on the raised edge of the tray and the cranial face supported by the adjustable stop, the machine was turned on, the tray was moved, a complete cut of the surface was made and then the machine was turned off. No slices were obtained but ground tissues, because of the low temperature of the block. The frozen block was taken to the photograph table and positioned with the freshly-cut side facing the camera. The surface was cleaned with $80 \%$ ethyl alcohol cooled to $20^{\circ} \mathrm{C}$, to remove the remaining frost and debris, and the photographic capture of the cut surface was made. Each cutting process involved measuring again the thickness of the cut and carefully positioning the block in the machine. The block was frozen again at $-20^{\circ} \mathrm{C}$ after 2 hours of work, preventing melting. The cutting process was performed until the entire cat was sectioned and photographed.

\section{Image Capture and Photography}

Artificial lighting under white light fluorescent lamps was used to photograph each sectioned surface with a Nikon D5500 (Nikon Corporation, Tokyo, Japan) digital camera equipped with a 55-300 lens. As the laboratory had several windows, the photographs were taken at night to avoid the effect of natural light. The camera was installed on a Beston 3560 tripod (Beston, Bogotá, Colombia) stand at a fixed distance and height from the metal table, so that the framing included the area of the cut surface of the block, and the plane of the cut surface was parallel to the framing plane of the photograph. After cutting, the frozen block was placed on 

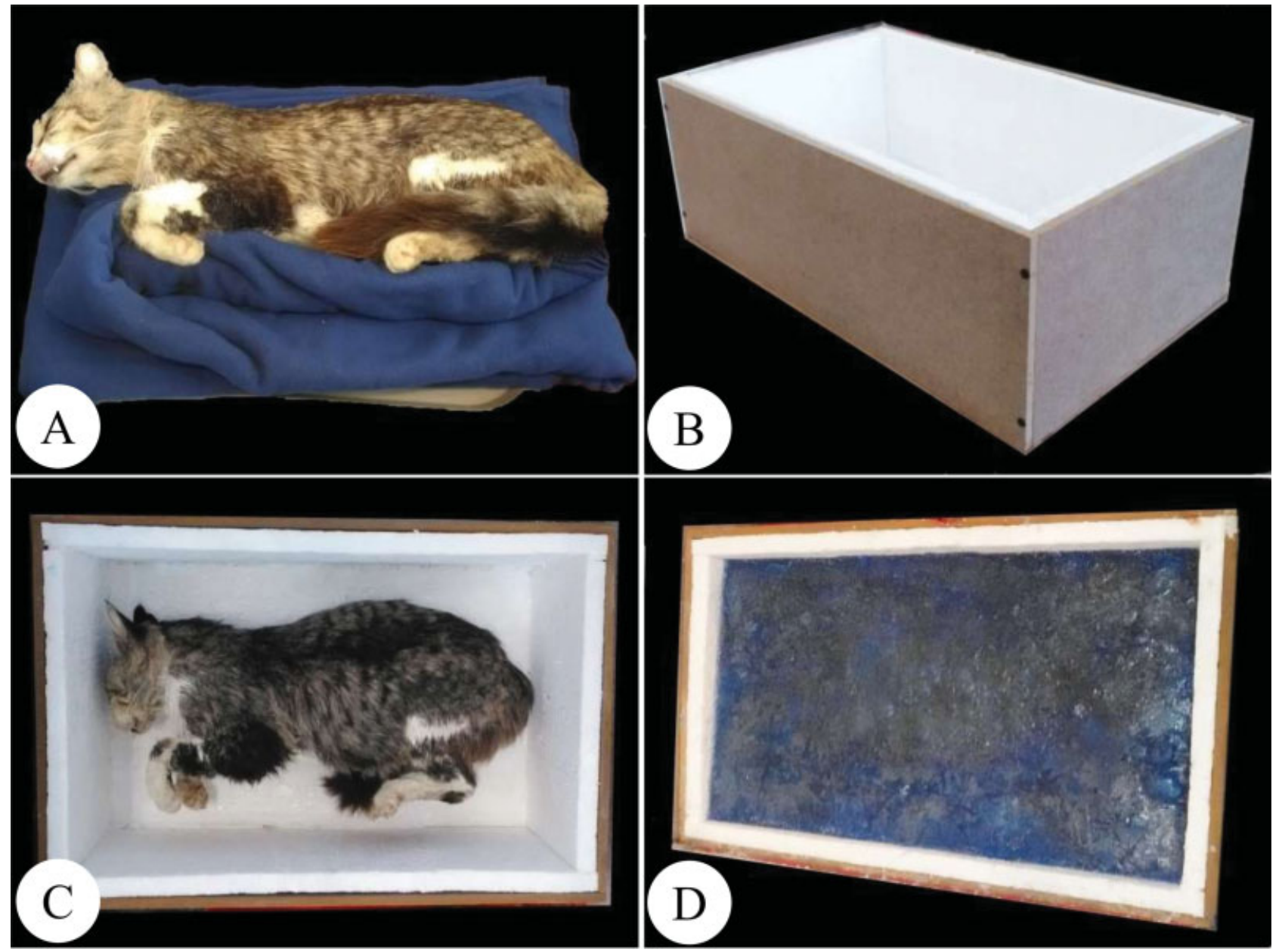

Fig. 1 Preparation of the cadaver. (A) Cadaver positioned with flexed limbs and tail. (B) Wooden box made with polystyrene cover. (C) Cadaver frozen and positioned inside the box before the embedding process. (D) Box with frozen block of embedded fluid with the cadaver inside.

the metal table in a predetermined position, the surface was cleaned as already mentioned, and the photographs were taken (automatic focus, aperture f 9 , ISO 400 , shooting speed 1/15). All of the photographs had the same technical and lighting characteristics, and were stored in the internal memory of the camera and in a computer.

\section{Computed Tomography and Magnetic Resonance Imaging Images}

The Universidad Nacional de Colombia provided 2 cadavers of adult male mongrel cats (approximate weight: $4 \mathrm{Kg}$, no age data available), one for scanning by $\mathrm{CT}$, and the other for scanning by MRI. The cadavers were preserved at $-20^{\circ} \mathrm{C}$ until usage. For the scanning procedures, the animals were thawed and placed in sternal recumbency with the limbs and neck in hyperextension. One of the cadavers was scanned by CT with a Philips Tomoscan AV (Philips Healthcare, Amsterdam, Netherlands) at $2 \mathrm{~mm}$ intervals, while the other cadaver was scanned by MRI with an Esaote Vet-MR 0.25 Tesla (Esaote, Genoa, Italy) at 3-mm intervals.

\section{Three-dimensional Modeling}

The modeling of the complete anatomy of a feline was made using Autodesk Maya, version 2017, (Autodesk Inc., San
Rafael, California, USA) and ZBrush, version 4R7 (Pixologic, Los Angeles, CA, USA) software. Three-dimensional objects for the structures and organs of the animal were created and then linked to form a complete 3D cat.

\section{Development of the Interactive Virtual Software}

An interactive virtual software was developed with the 3D cat and images library using Unity, version 5.4 (Unity Technologies, San Francisco, CA, USA). For the image library, 30 representative equivalent images from each technique were selected, for a total of 90 images of the entire body of the animal. All of the images were edited to match the same resolution characteristics (1,024 × 1,024 pixels), filter, background, and labels. The CT and MRI images were transformed from the DICOM format to JPG.

\section{Results}

\section{Sectioning and Photography}

A total of 178 cross-sectional photographs of the frozen block of the entire cat cadaver were obtained with 2.5 -mm intervals. The images were converted to JPG files (file size 13.4 $\mathrm{MB} /$ image, total file size $2.3 \mathrm{~GB}$ ) with a resolution of $6,000 \times 4,000$ pixels ( - Table 1 ). 
Table 1 Quantity and characteristics of the different types of images obtained

\begin{tabular}{|l|l|l|l|l|l|}
\hline Images Type & Quantity & Intervals & Resolution (Pixels) & Size & Format \\
\hline Magnetic resonance imaging & 135 & $3 \mathrm{~mm}$ & $512 \times 512$ & $146 \mathrm{~KB}$ & $\mathrm{DICOM}$ \\
\hline Computed tomography & 330 & $2 \mathrm{~mm}$ & $512 \times 512$ & $329 \mathrm{~KB}$ & $\mathrm{DICOM}$ \\
\hline Photographs & 178 & $2.5 \mathrm{~mm}$ & $6,000 \times 4,000$ & $13.4 \mathrm{MB}$ & $\mathrm{JPG}$ \\
\hline Total & 643 & & & & \\
\hline
\end{tabular}

Abbreviations: DICOM, digital imaging and communications; JPEG, joint photographic experts group.

The photographs showed the anatomy of the animal with detail. All of the organs and many of the structures throughout the body could be identified and differentiated. The color of the photographs facilitated the differentiation of a great variety of structures - for example, the gray and white matter ( - Fig. 2A). The continuity of structures, such as of the trachea, of the cranial vena cava, of the aorta, and of the spinal cord, could be appreciated. Differentiated structures included individual muscles (i.e., the supraspinatus, the triceps brachii, and the quadriceps femoris muscles), bones (i.e., the femur, the ulna, and the sternum), and organs (i.e., the mucosa of the stomach, the parenchyma of the liver, and the renal marrow and cortex). Some small (i.e., the meninges, and the skin) and large structures (i.e., coxal, intestines) could also be recognized, but others, such as small blood vessels, were not discernible (-Fig. 2 ).

\section{Computed Tomography and Magnetic Resonance Imaging Images}

A total of $135 \mathrm{MRI}$ images were obtained at 3-mm intervals (file size $146 \mathrm{~KB}$ /image, total file size $20 \mathrm{MB}$ ) with a resolution of $512 \times 512$ pixels. The images showed in detail several anatomical structures and organs of the cat (-Fig. 3). The different gray gradations allowed the differentiation between tissue types (muscle, bone, air, and fluids), cavities (i.e., the frontal sinus), solid organs (i.e., the liver, the lungs) and hollow organs (i.e., the stomach). The continuity of structures such as of the trachea and of the spinal cord was easily discerned. Among others, some of the structures that were easily recognized included: muscle groups (i.e., the sublumbar muscles), bones (i.e., the jawbone, the ribs, the sternum), the interior of some organs (i.e., lumen of the bowel, parenchyma of the liver, interior of the eye), structures of medium (i.e., vertebral bodies) and large dimensions (i.e., intestines). No small structures, such as blood vessels of medium and small caliber, could be identified. In nervous tissues, the gray matter was not distinguished from the white matter.

A total of $330 \mathrm{CT}$ images were obtained using 2-mm intervals (file size $329 \mathrm{~KB} /$ image, total file size $108 \mathrm{MB}$ ) with a resolution of $512 \times 512$ pixels. The gray scales allowed the differentiation of soft tissue structures with poor detail (i.e., the liver, the stomach), but muscles could not be differentiated. Spaces occupied by bone tissue and air were clearly delineated. As with the MRI images, the continuity of structures, such as of the trachea and of the spinal cord, could be clearly demarcated. Other easily identified structures included air cavities (i.e., acoustic meatus, segmental bronchi, gas in the intestine), bones (i.e., the radius, the femur, the sternum, the skull, the ribs), organs (i.e., the heart, the lungs), and structures of medium (i.e., vertebrae) and large size (i.e., coxal). No small structures, such as blood vessels of medium or small caliber, could be identified. In nervous tissue, the gray matter was not differentiated from the white matter. In the abdominal cavity, the organs were not easily recognized - for example, the kidney and the spleen could not be identified (-Fig. 4).

\section{Three-dimensional Modeling}

A total of $\sim 418$ structures were modeled for the skeletal, muscular, circulatory, nervous, respiratory, digestive, urinary, and integumentary systems (-Fig. 5).

\section{Development of the Interactive Virtual Software}

Two user interfaces were created, one for viewing organ systems of the 3D cat, another for viewing selected images. A panel with icons for the visualization of every organ system of the 3D cat was provided. The systems are activated by clicking on the icon of each organ system and are deactivated by clicking on the button again. Any system can be activated individually, or several systems can be activated at the same time. The images can be zoomed in and out, and can be moved and rotated according to the interest of the user of the 3D cat panel. Additionally, several structures of different systems stand out and their names are indicated when the mouse is scrolled over them (-Fig. 6). The muscular system structures have an additional command that allows them to fade and reappear for easier visualization.

The interface for displaying the image library has an initial screen that shows a cat with several points for the plane of interest. By selecting a point, the user gains access to the next screen, which shows the image of the corresponding CT slice (by default), and a panel with buttons to compare every image for any technique (MRI, photograph, or CT). When scrolling the mouse over the selected image, the names of some of the structures are disclosed (-Fig. 7).

A demo of the software is shown in a published YouTube video (https://www.youtube.com/watch?v=Qr4XazpMCEg) and a link is provided in it to allow free downloading.

\section{Discussion}

The preparation of a cadaver is crucial to obtain an animal without alterations in anatomy, shape, and color. Embedding was conducted in several stages to avoid air chambers that could change the shape of the animal, ${ }^{16,18,23}$ and fixation 

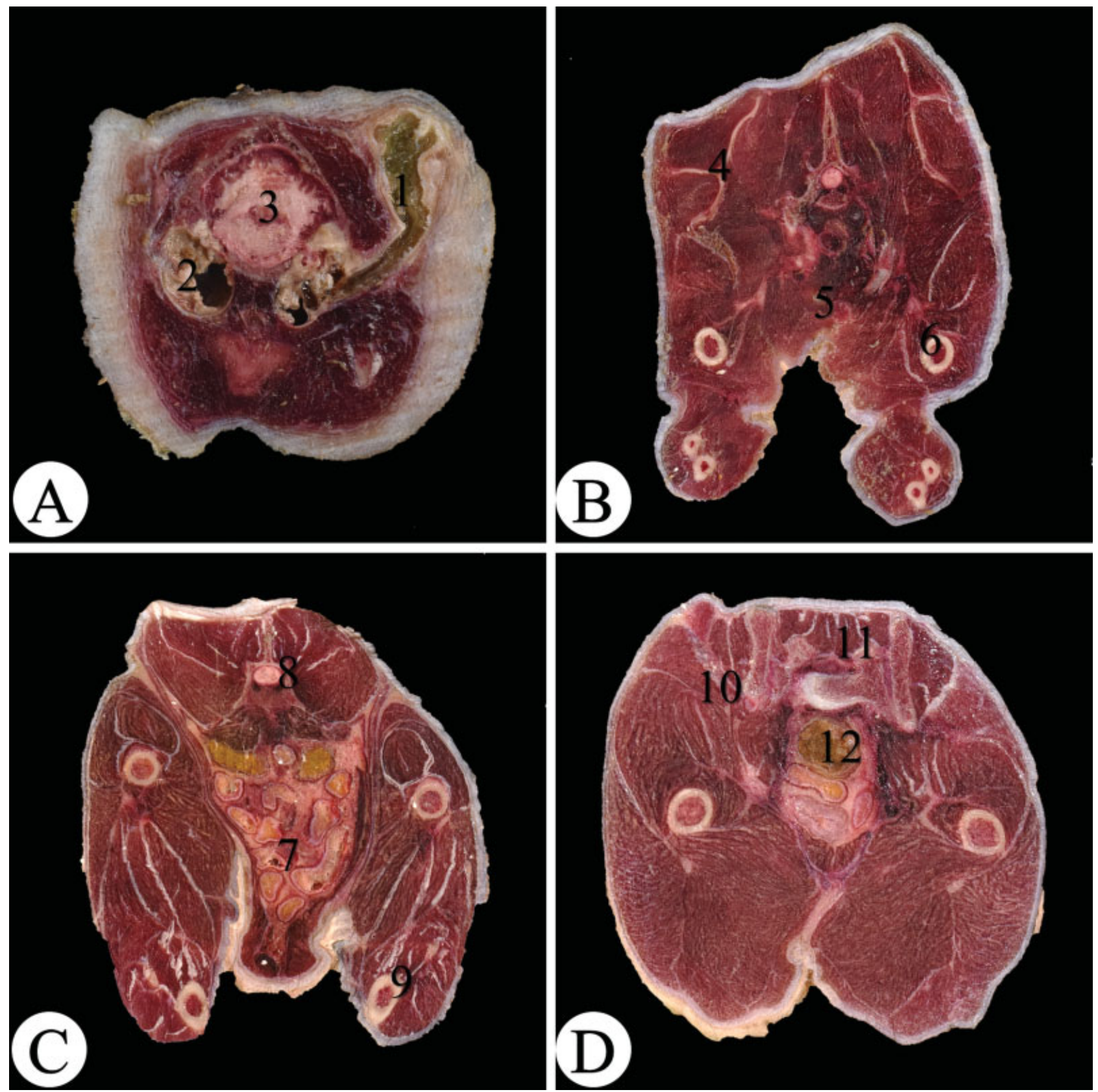

Fig. 2 Photographs of cross-sections of a cat cadaver. (A) Cranial region at the level of the brain, (B) thoracic region at the level of the second thoracic vertebra, (C) abdominal region at level of the kidneys, and (D) the pelvic region at the level of the coxal joint. (1) Brain, (2) jawbone, (3) masseter muscle, (4) humerus, (5) ulna and radius, (6) scapula, (7) right kidney, (8) stomach, (9) liver, (10) femur, (11) coxal, (12) rectum.

avoided alternations in the colors of the tissues. ${ }^{22,23}$ Heparin could not be injected previously to death in the cat we have used; therefore, no substances were injected to highlight the blood vessels, because the presence of clots in the circulatory system may prevent the movement of any perfused substance and cause an obstruction that could alter the anatomy. ${ }^{16,18,19}$

Proper sectioning requires freezing the cadaver at the lowest possible temperature to obtain flat uniform surfaces and avoid quick thawing. Although similar projects have used freezing temperatures of $-80^{\circ} \mathrm{C}^{16,18,19,22,23}$ in our case the freezer could only attain temperatures of $-20^{\circ} \mathrm{C}$. Despite this limitation, there were no problems with sectioning due to thawing of the block, suggesting that $-20^{\circ} \mathrm{C}$ can also be used as an alternative temperature. To avoid rapid thawing, a layer of polystyrene was used to wrap and isolate the block, the sectioning was performed at night to avoid daytime higher temperatures, and the surface was cleaned with $70 \%$ alcohol cooled to $-20^{\circ} \mathrm{C} .^{16,18,19}$

For image capturing, the body must be in an adequate position so that the images recreate the actual anatomy. ${ }^{19,21}$ For the photographs, a quilted blanket was used to avoid deformation of the area on which the cadaver was lying. Because the cutting area of the bandsaw was limited, the position was chosen to occupy the smallest space possible that would be totally covered by the frozen block and by the width of the cutting area. 

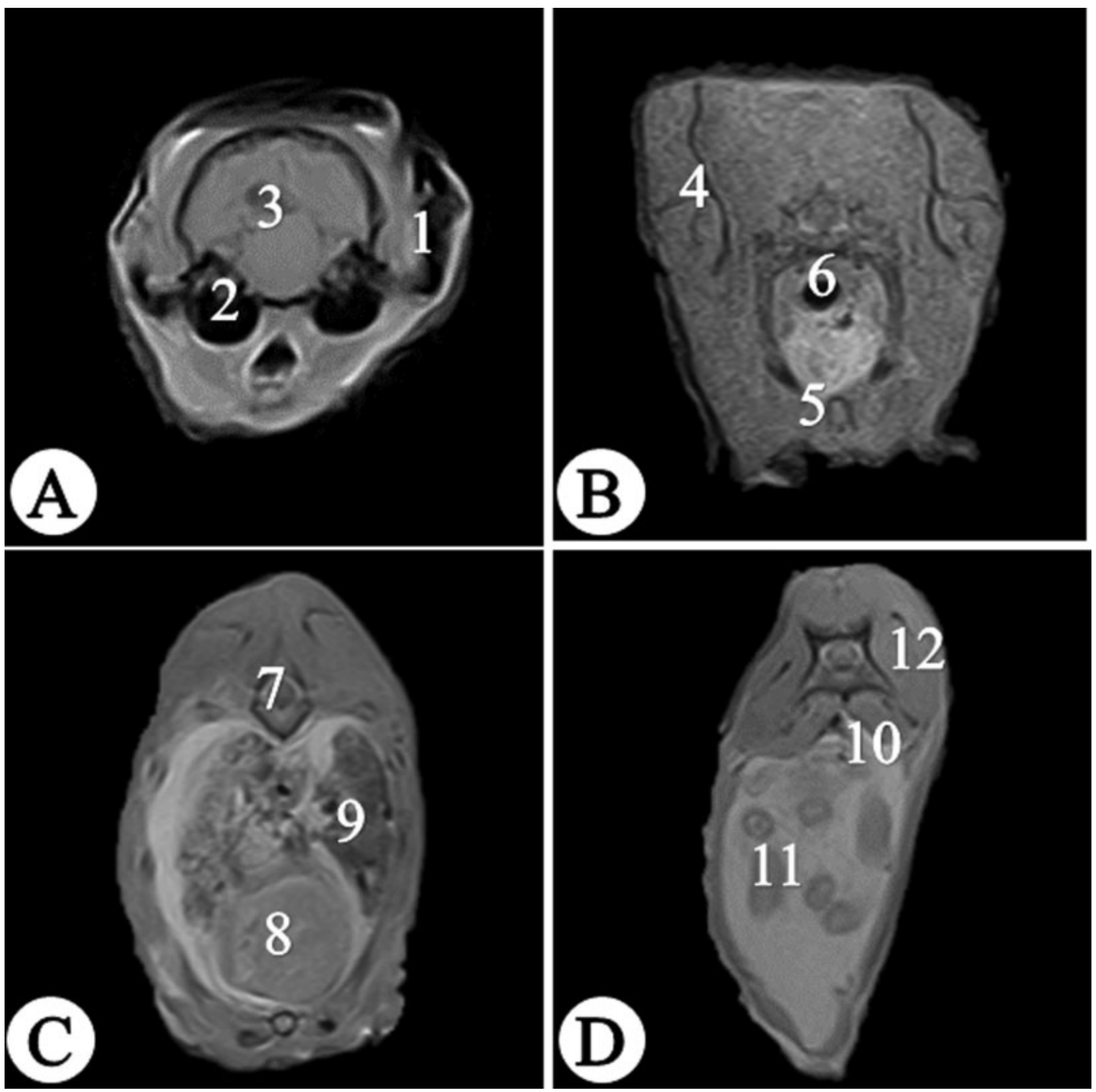

Fig. 3 Magnetic resonance imaging of (A) cranial region at the level of the frontal sinus, (B) thoracic region at the level of the second thoracic vertebra, and the region of the abdomen (C) at the level of the liver and (D), at the level of the last lumbar vertebrae. (1) Frontal sinus, (2) nasal cavity with turbinates, (3) jawbone, (4) scapula, (5) trachea, (6) sternum, (7) liver, (8) right lung, (9) vertebral body, (10) sublumbar muscles (10), intestines, (12) epaxial muscles.

For absolute correspondence among the images, all of the captions by every technique (MRI, CT and digital photographs) should be taken on the same animal and positions. $^{18,21,22}$ We have obtained images with each method from a different animal, in different positions, and at different section intervals. A major limitation was the availability of CT and MRI machines for veterinary use in the country. The only available machines were in another city, so there was no possibility of using the same animal due to the difficulty of transporting a cadaver from one city to another. Additionally, it was not possible to coordinate the two scanning processes for the same day with the same animal. Even using the same cadaver in similar projects report slight changes in position between different types of images. ${ }^{6,18,19}$ The images were taken at different intervals due to the technical restrictions of the MRI and CT machines. Although there was no complete correspondence between the three techniques, a group of images equivalent to each other was obtained, which also allowed comparisons between the methods used.

The characteristics of the digital camera and of the lighting are decisive factors for the proper quality of the photographs. The capacity of the camera determines the resolution of the photograph, which is determined by the number of pixels. In the present study, a 24.2 megapixel camera was 

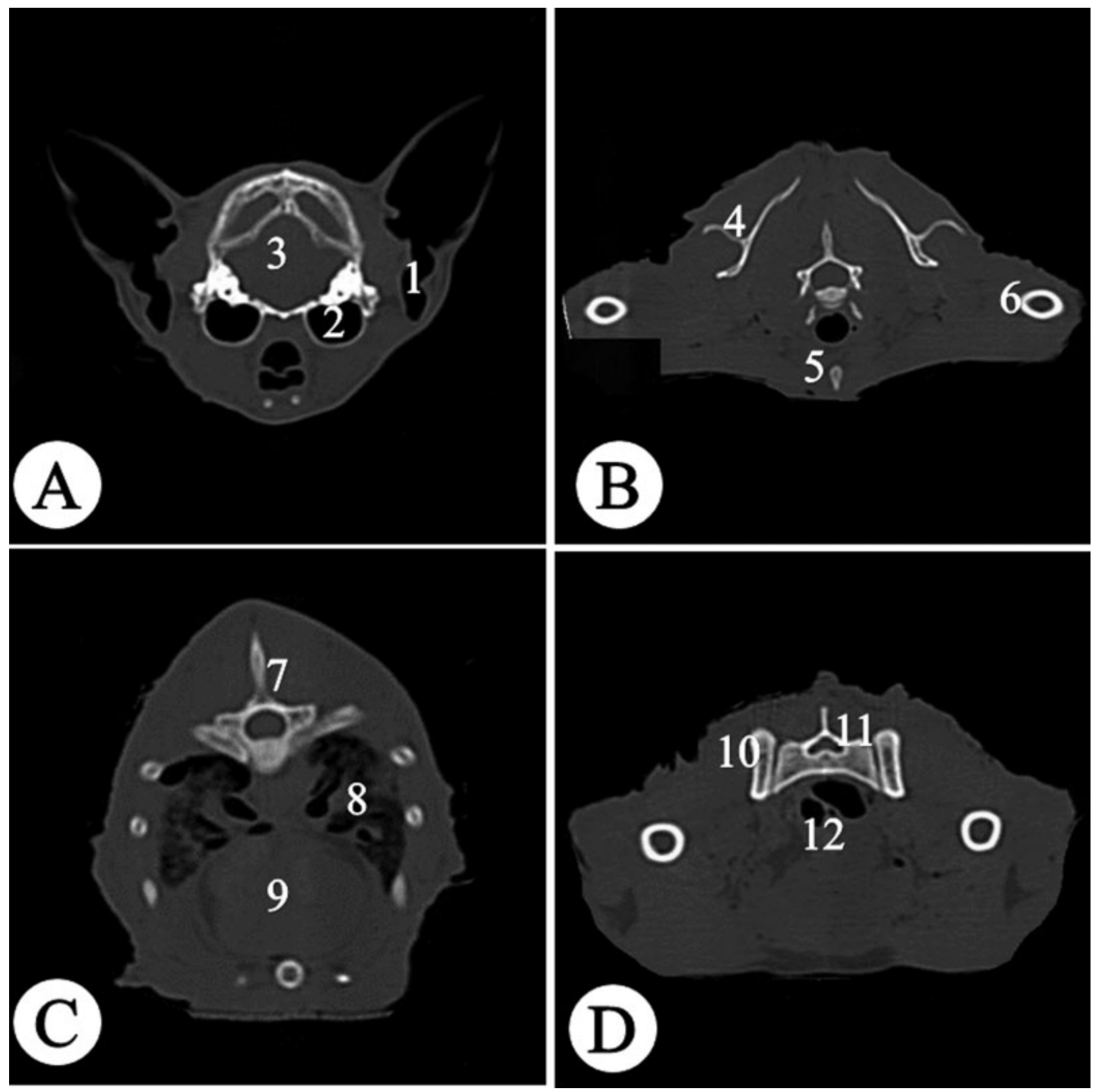

Fig. 4 Computed Tomography images of (A) cranial region at the level of the tympanic bulla, thoracic region (B) at the level of the second thoracic vertebra, (C) at the level of the heart, and (D) pelvic region at the level of the coxal joint (D). (1) Acoustic meatus, (2) tympanic bulla, (3) encephalon, (4) scapula, (5) sternum, (6) humerus, (7) thoracic vertebra, (8) heart, (9) left lung, (10) femur, (11) coxal, and (12) rectum.

used, providing an excellent resolution of the photographs. ${ }^{16,18,19,23}$ Because the lighting should be constant and unchanged, the pictures were taken at night under constant artificial light. ${ }^{21,22}$

The software developed in the present study has two interfaces for easier navigation, one to explore the 3D cat and another to explore the image library. The interface approach for the 3D cat facilitates visualization and precise scene composition. The user can create any scene of interest, with the possibility of exploring the whole cat, a certain region of interest, or a particular structure. Text labels provide the name of the structure and the highlighting in color facilitates its recognition and location. The interface of the image library allows comparing equivalent images by CT, MRI and digital photographs, and rapid changes between planes to facilitate the understanding of sectional anatomy.

Compared with interactive virtual models, printed images have the limitation that they are static and not expandable, the labeling is often limited or incomplete, the number of views is limited, and the spatial relationships are difficult to understand. ${ }^{4,5}$ Interactive virtual models reduce these limitations and become a valuable educational tool. If this type of interactive virtual resource is incorporated into the curricula, it can help to create a user-controlled learning experience that is customized and adapted to different types of learners. $2,5,21$ 

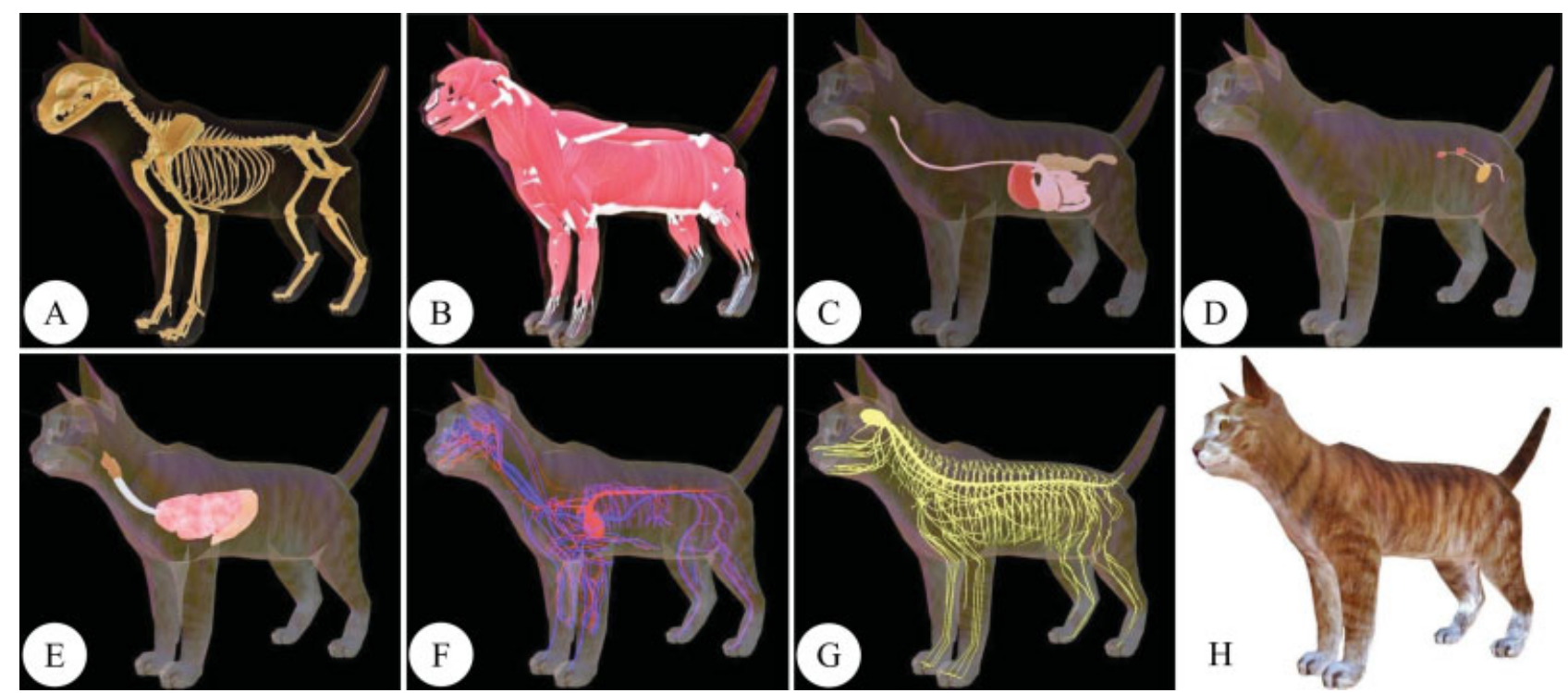

Fig. 5 Three-dimensional cat model of the anatomical systems. (A) Skeletal, (B) muscular, (C) digestive, (D) urinary, (E) respiratory, (F) circulatory, $(\mathrm{G})$ nervous systems, and $(\mathrm{H})$ skin and exterior model of the cat.

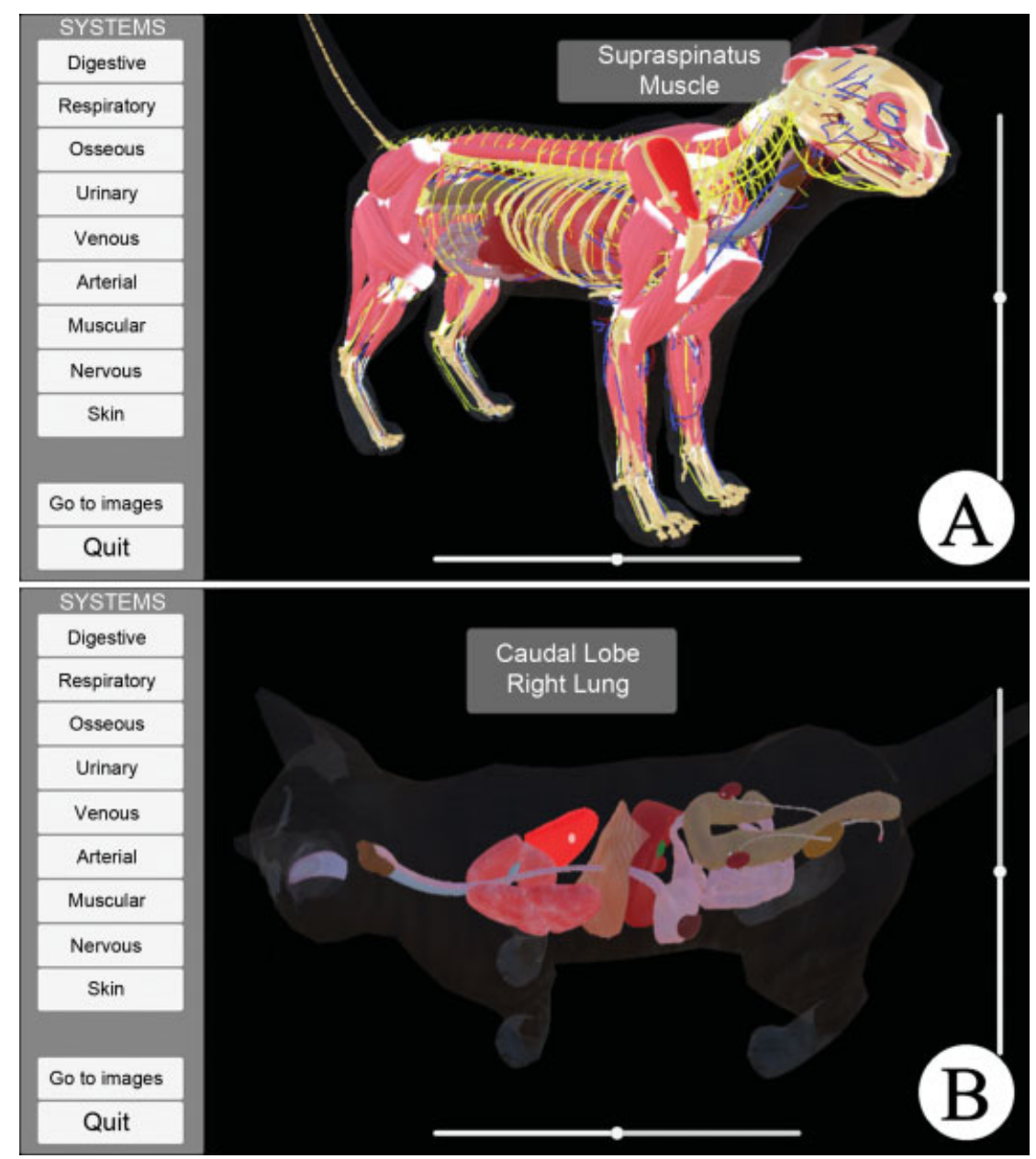

Fig. 6 User interface for the visualization of anatomical structures and systems. (A) Three-dimensional cat with zoom in, rotation, several organic systems activated and some hidden muscles, with the pointer indicating the infraspinatus muscle. (B) Three-dimensional cat with zoom in, and rotation, with the digestive, urinary and respiratory systems activated, with the pointer on the cranial lobe of the right lung. 

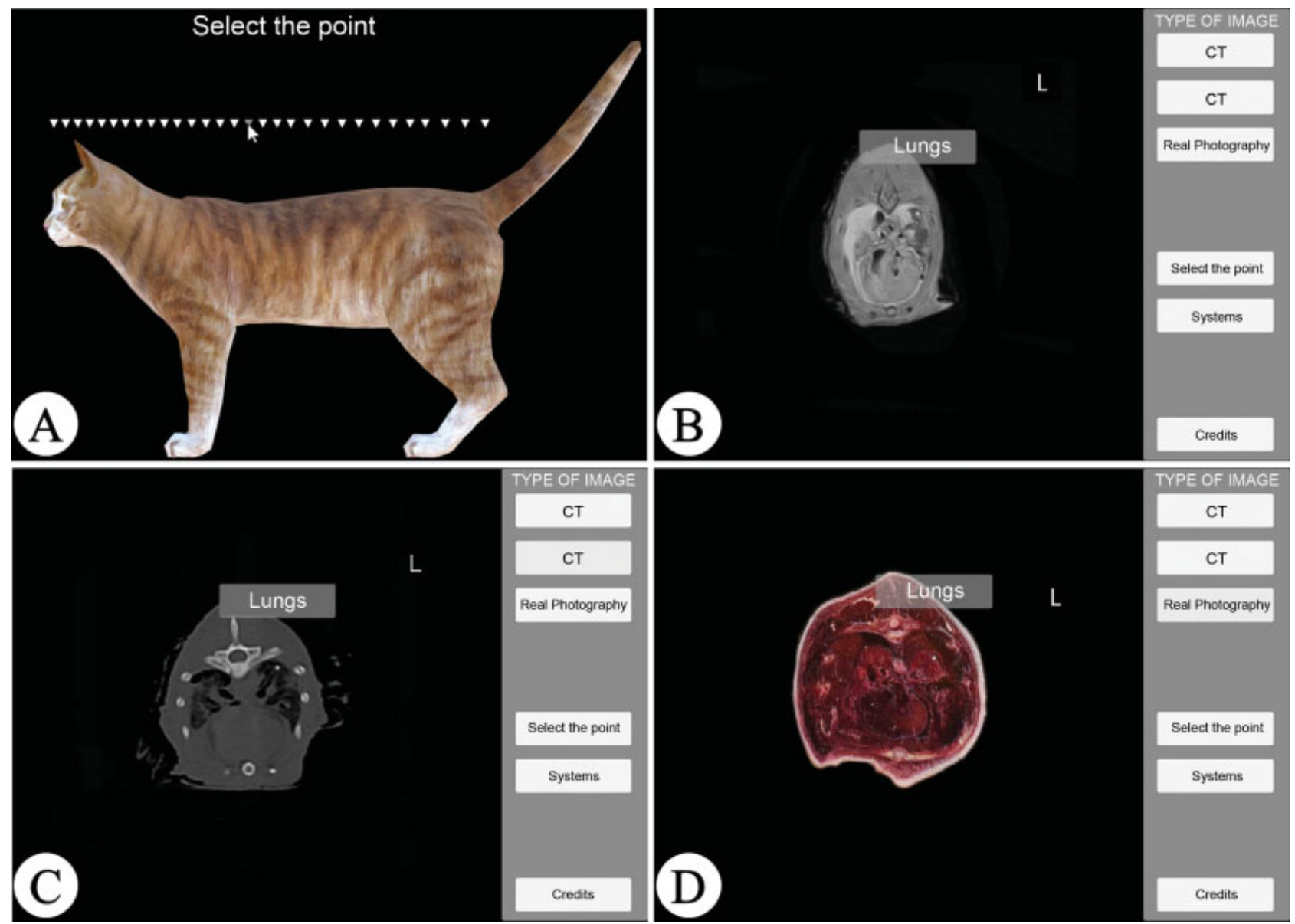

Fig. 7 User interface for displaying the image library. (A) Screen of selection of the plane of interest (thorax region). Screen with magnetic resonance imaging (B) and computed tomography (C) of the selected region with the pointer located above the lungs. (D) Screen with a photograph of a transverse section of the thorax region with the pointer located on the lungs.

This 3D virtual model software of the cat will simplify learning anatomy in veterinary education.

\section{Conclusions}

A thorough knowledge of veterinary anatomy is an important prerequisite for a proper interpretation of modern medical images. When interpreting images, knowledge of the dimensions and of the localization of anatomical structures is essential. For veterinary students and practitioners, it is difficult to envision 3D objects in space. The use of 3D virtual models can facilitate the interpretation of modern techniques of body imaging. In addition, these models reduce the difficulties of visualizing some anatomical structures, as occurs with 2D images.

There is a need to shorten the learning curve for students in the acquisition of basic anatomical knowledge. Threedimensional virtual models could help to achieve this goal, allowing recognizing structures, exploring the body, making topographical relationships, simplifying structures, isolating regions or body parts with the possibility of easily changing the point of view.

Future studies must evaluate the efficacy of the software as a learning tool in veterinary anatomy, as well as students and faculty perceptions.

\section{Conflicts of Interest}

The authors have no conflicts of interest to declare.

\section{Acknowledgments}

We wish to thank the Comité para el Desarollo de la Investiacíon de la Universidad de Atioquia (CODI-UdeA) for the funding grant to develop the present work. We also wish to thank professor Hugo Gutiérrez, from Universidad Nacional de Colombia, for all the collaboration to this work.

\section{References}

1 Lewis TL, Burnett B, Tunstall RG, Abrahams PH. Complementing anatomy education using three-dimensional anatomy mobile software applications on tablet computers. Clin Anat 2014;27 (03):313-320

2 Nicholson DT, Chalk C, Funnell WRJ, Daniel SJ. Can virtual reality improve anatomy education? A randomised controlled study of a computer-generated three-dimensional anatomical ear model. Med Educ 2006;40(11):1081-1087

3 Jang HG, Chung MS, Shin DS, et al. Segmentation and surface reconstruction of the detailed ear structures, identified in sectioned images. Anat Rec (Hoboken) 2011;294(04):559-564

4 Allen LK, Bhattacharyya S, Wilson TD. Development of an interactive anatomical three-dimensional eye model. Anat Sci Educ 2015;8(03):275-282 
5 Nowinski WL, Thirunavuukarasuu A, Volkau I, et al. A new presentation and exploration of human cerebral vasculature correlated with surface and sectional neuroanatomy. Anat Sci Educ 2009;2(01):24-33

6 Geyer LL, Schoepf UJ, Meinel FG, et al. State of the art: iterative CT reconstruction techniques. Radiology 2015;276(02):339-357

7 Henn JS, Lemole GM Jr, Ferreira MA, et al. Interactive stereoscopic virtual reality: a new tool for neurosurgical education. Technical note. J Neurosurg 2002;96(01):144-149

8 Martinsen S, Jukes $\mathrm{N}$. Towards a humane veterinary education. J Vet Med Educ 2005;32(04):454-460

9 Preece D, Williams SB, Lam R, Weller R. "Let's get physical": advantages of a physical model over 3D computer models and textbooks in learning imaging anatomy. Anat Sci Educ 2013;6 (04):216-224

10 Leung KK, Lu KS, Huang TS, Hsieh BS. Anatomy instruction in medical schools: connecting the past and the future. Adv Health Sci Educ Theory Pract 2006;11(02):209-215

11 Shin DS, Chung MS, Park JS, et al. Three-dimensional surface models of detailed lumbosacral structures reconstructed from the Visible Korean. Ann Anat 2011;193(01):64-70

12 Park JS, Chung MS, Hwang SB, Lee YS, Har DH. Technical report on semiautomatic segmentation using the Adobe Photoshop. J Digit Imaging 2005;18(04):333-343

13 Segars WP, Mahesh M, Beck TJ, Frey EC, Tsui BM. Realistic CT simulation using the 4D XCAT phantom. Med Phys 2008;35(08): 3800-3808

14 Segars WP, Tsui BM, Frey EC, Johnson GA, Berr SS. Development of a 4-D digital mouse phantom for molecular imaging research. Mol Imaging Biol 2004;6(03):149-159
15 Lauridsen $\mathrm{H}$, Hansen $\mathrm{K}$, Wang T, et al. Inside out: modern imaging techniques to reveal animal anatomy. PLoS One 2011;6(03): e17879

16 Spitzer V, Ackerman MJ, Scherzinger AL, Whitlock D. The visible human male: a technical report. J Am Med Inform Assoc 1996;3 (02):118-130

17 Schiemann T, Freudenberg J, Pflesser B, et al. Exploring the Visible Human using the VOXEL-MAN framework. Comput Med Imaging Graph 2000;24(03):127-132

18 Zhang SX, Heng PA, Liu ZJ. Chinese visible human project. Clin Anat 2006;19(03):204-215

19 Park JS, Chung MS, Hwang SB, Shin BS, Park HS. Visible Korean Human: its techniques and applications. Clin Anat 2006;19(03): 216-224

20 Paul Segars W, Tsui BM. MCAT to XCAT: The Evolution of 4-D Computerized Phantoms for Imaging Research: Computer models that take account of body movements promise to provide evaluation and improvement of medical imaging devices and technology. Proc IEEE Inst Electr Electron Eng 2009;97(12): 1954-1968

21 Dogdas B, Stout D, Chatziioannou AF, Leahy RM. Digimouse: a 3D whole body mouse atlas from $\mathrm{CT}$ and cryosection data. Phys Med Biol 2007;52(03):577-587

22 Böttcher P, Maierl J. Macroscopic cryosectioning: a simple new method for producing digital, three-dimensional databases in veterinary anatomy. Anat Histol Embryol 1999;28(02): 97-102

23 Park HS, Shin DS, Cho DH, Jung YW, Park JS. Improved sectioned images and surface models of the whole dog body. Ann Anat 2014; 196(05):352-359 